\title{
Entrevista a María Gracia Cariola: una mirada al proyecto de ley que crea el permiso postnatal parental
}

Este comentario, junto a los documentos que analiza, está disponible en www.anuariocdh.uchile.cl

Por Verónica Undurraga ${ }^{1}$

María Gracia Cariola es abogada de la Pontificia Universidad Católica de Chile, Máster in Comparative Jurisprudence de la Universidad de Nueva York. Fue presidenta de la Comisión Asesora Presidencial Mujer, Trabajo y Maternidad convocada por el Presidente Sebastián Piñera. Actualmente es Gerente General de Inversiones MC\&MC. S.A., Directora de Pathfinder, Directora de Feria de Osorno, Directora de Empresas La Polar, Directora de ESSAL y Vicepresidenta de la Sociedad Nacional de Agricultura (SNA). Es consejera de Comunidad Mujer y de Endeavor.

A la Comisión Asesora Presidencial Mujer, Trabajo y Maternidad (la Comisión) se le encomendó como objetivo: elaborar una propuesta para promover la incorporación de la mujer al mundo del trabajo, fortalecer su autonomía económica, mejorar la distribución de responsabilidades y costos de maternidad y cuidado infantil entre hombres y mujeres, mejorar la compatibilización de familia y trabajo para hombres y mujeres, y fortalecer la familia y proteger la maternidad. La Comisión trabajó durante tres meses, al término de los cuales entregó un informe al Presidente de la República ${ }^{2}$. Con fecha 28 de febrero de 2011 el gobierno presentó al Senado el mensaje con el que inicia un proyecto de ley que crea el permiso postnatal parental ${ }^{3}$.

Verónica Undurraga: Un aspecto que me Ilama la atención sobre el debate público en torno al postnatal es que pareciera que los costos y los beneficios asociados a la extensión del postnatal son difíciles de prever. Da la impresión que no se tiene certeza de cuánto puede afectar la contratación de mujeres ni la medida en que esta política traerá los beneficios sociales relacionados con el apego y la salud de los niños y niñas. ¿Comparte usted el juicio que esta es una política que se debió adoptar en un contexto de incertidumbre? ¿Cuál es su percepción sobre la información que tuvo a su disposición la Comisión para trabajar en la propuesta y el gobierno para formular el proyecto?

María Gracia Cariola: Durante todo el periodo que trabajamos en la Comisión me sorprendió la falta de estudios cuantitativos y cualitativos, no solo en Chile, sino también en otras partes del mundo, respecto a las consecuencias que puede tener la implementación de este tipo de políticas públicas. Es impresionante lo poco que se miden los impactos de las políticas públicas. No sé si

Directora del Programa de Mujeres y Derechos Humanos, abogada, diplomada en Instituciones Modernas de Derecho de Familia, Máster of Laws (LL.M.) Columbia University N.Y., candidata a doctora en derecho por la Universidad de Chile.

Agradecemos especialmente la asistencia brindada por la ayudante Aurora Muñoz, estudiante de Derecho de la Universidad de Chile, en la preparación de esta entrevista

2 El informe de la Comisión puede ser leído en http://www.comisionmujertrabajoymaternidad.cl/index.php?option=com_c ontent\&view=article\&id=30\&Itemid=10 (última visita 26 de abril de 2011)

3 El proyecto de ley puede ser leído en http://www.gob.cl/media/2011/03/ley_posnatal.pdf (última visita 26 de abril de 2011). 
es porque estos no son los temas que más se miden, pero lo que había disponible no daba una luz total. Lo que sí se había medido es que en los países en los cuales no existían políticas de protección a la maternidad y éstas se introducían, tenían el efecto favorable de incrementar la fuerza de trabajo femenina. Esto sucedía porque una cantidad de mujeres que nunca había salido a trabajar lo empieza a hacer cuando siente que está más protegida. También existen una serie de estudios científicos y médicos, por ejemplo sobre lactancia y apego, en los que hay un state of the art (estado del arte), pero muchos de ellos dicen cosas contradictorias. Además el conocimiento va avanzando, se sigue investigando, entonces es difícil ser tajante. Una conclusión es válida ahora, pero puede no ser válida después. Otro aspecto que hay que tomar en cuenta es que, si bien las políticas públicas se adoptan basadas en la información existente, los estudios suelen ser muy específicos y se refieren a aspectos distintos. Entonces, por ejemplo, la Organización Mundial de la Salud puede recomendar favorecer la lactancia materna en los primeros seis meses, basándose en una multiplicidad de estudios sobre los beneficios del apego, la estimulación, la realidad de la desnutrición en algunas áreas, los beneficios de la lactancia materna en términos de higiene, etc. La recomendación de la política pública en ese ejemplo se basó en investigaciones, pero implicó asumir una generalización de las conclusiones de esos estudios a veces para realidades en que las conclusiones de esos estudios no serían pertinentes. A diferencia de la Comisión Asesora para la Reforma Previsional y del Consejo de Trabajo y Equidad, que tuvieron más tiempo y recursos para encargar una serie de estudios, nosotros no pudimos encargar ninguno. En la Comisión sabíamos que estábamos tratando sobre una materia en que no había información que fuera del todo acabada. Hay información, pero no es una información que permita concluir tajantemente vamos por acá, esto lo que hay que hacer. En el tema del impacto de este tipo de políticas en la contratación de mujeres, ahí sí que existe poco. Además este impacto es muy difícil de medir, porque en general estas políticas no se implementan solas, hay una serie de otras políticas que se implementan junto con ellas y es muy difícil aislar la variable. Por eso y dada esa incertidumbre es que una recomendación de la Comisión fue que hay que ser lo más prudente posible en la implementación de una extensión del postnatal.

\section{$V U:$ ¿Qué significa ser prudente en este tema?}

MGC: Nosotros lo entendíamos como implementarlo progresivamente, para poder ir midiendo el impacto y sobre la base de esas mediciones haber podido seguir tomando decisiones e ir dirigiendo más apropiadamente la implementación de la política pública.

\section{VU: ¿Cómo evalúa el aporte que hicieron los/as especialistas representantes de la sociedad civil que fueron convocados/as por la Comisión? ¿Cree que fue suficiente? ¿Se consultó a todas las personas que debía consultarse?}

MGC: Yo creo que lo que más hicimos fue oír, convidando a todos los que pensamos tenían que aportar y recibiendo a toda la gente que quiso opinar. Por parte de la sociedad civil hubo gran interés en participar. Me llamó la atención el poco interés de los parlamentarios, salvo los que tenían una agenda muy marcada con el tema.

\section{$V U:$ ¿Y el nivel de información qué manejaban?}

MGC: Los que tenían una agenda marcada con el tema manejaban información, pero era una información médica, eran casi todos doctores. Otro tipo de información, no. A mí se me hizo evidente el propósito de estas comisiones y lo fértil que pueden ser, precisamente dada la poca información que existe en el mundo parlamentario respecto de estos temas. Las comisiones sirven como puente entre quienes sí tienen conocimientos, los expertos, quienes trabajan en los centros 
de estudios y en las universidades y quienes toman decisiones. De alguna manera el conocimiento se hace más visible. Si se toma después en cuenta o no es otra cosa.

VU: ¿No cree usted que habría sido mejor, para evitar el riesgo de profundizar más el problema de la baja participación laboral femenina, gastar los recursos destinados a la ampliación del postnatal en masificar, por ejemplo, la oferta de salas cunas y jardines infantiles?

MGC: Bueno, la Comisión, a propósito de esta idea de ampliar el postnatal y respondiendo al mandato que nos dio el Presidente Piñera, hizo un estudio completo del régimen de protección de la maternidad. Y la gran conclusión que sacó la Comisión fue que en un país como Chile lo primero que había que hacer era aumentar la cobertura y que aumentar la cobertura no implicaba solamente los permisos y subsidios, sino también el cuidado infantil. Ahí el gobierno de la Presidenta Bachelet hizo un gran trabajo. Es impresionante cómo se ha aumentado la cobertura de salas cunas. Yo creo que falta todavía avanzar en dos sentidos. El primero es que el aumento de cobertura debe darse de una manera más informada en relación con la demanda, es decir, identificar dónde tienen que estar estas salas cunas para que efectivamente sean usadas y demandadas y que no se produzca una superposición de oferta en algunas partes y falta de oferta en otros lugares. El segundo es que falta alcanzar a un segmento importante de la población que no son los más vulnerables -porque estos sí están cubiertos por el programa Chile Crece Contigo- que no tiene los medios para pagar una sala cuna privada y que tampoco trabaja en lugares en que el empleador esté obligado a dar el servicio. Se trata básicamente de personas de clase media, en donde hay una demanda insatisfecha importante.

VU: El artículo 203 del Código del Trabajo establece la obligación para los empleadores que tienen 20 o más mujeres contratadas de ofrecer servicio de sala cuna. Hay distintas formas de cambiar esa norma para que deje de afectar la empleabilidad de las mujeres. Una es que la norma no distinga entre trabajadores mujeres y hombres, de manera que no se cargue exclusivamente a la mujer con los gastos asociados a la crianza, y la otra es que las salas cunas pasen a ser financiadas totalmente por el Estado o bien o parcialmente por el Estado con copago de los padres. ¿Cuál es su opinión al respecto?

MGC: En la Comisión teníamos claro que la obligación de proveer salas cunas del artículo 203 del Código del Trabajo afecta la empleabilidad de las mujeres. Como está ahora evidentemente no es está bien. Yo y muchos en la Comisión éramos inicialmente de la idea de que el gasto de sala cuna fuera compartido entre hombres y mujeres y pensábamos que era fácil de implementar, simplemente diciendo que la obligación existía cuando se contratara 20 o más trabajadores, incluyendo hombres y mujeres. Pero el problema es que una gran cantidad de empresas chilenas no tienen la capacidad de solventar ese nivel de costo en salas cunas, que es muy alto. No son capaces y a la larga lo que pasa es que bajan el salario. Además una reforma de este tipo, que parece fácil en teoría, es difícil de implementar en la práctica. Llegamos a la conclusión en que tenía que ser un sistema en que el Estado pagara el total del costo para los más vulnerables y que para el resto hubiera un aporte parcial del Estado y un copago a cargo de la empresa y de las trabajadoras y los trabajadores.

VU: En el proyecto de gobierno no se incluye la reforma al artículo 203 del Código del Trabajo.

MGC: No, y entiendo que fue una gran pelea que dieron las ministras del Sernam y del Trabajo para que se incluyera. 


\section{VU: ¿Y no se incluyó por razones presupuestarias?}

MGC: Yo creo que por razones presupuestarias no se incluyó.

VU: De acuerdo al informe de la Comisión Asesora Presidencial Mujer, Trabajo y Maternidad, uno de los principios que orientó el trabajo y las propuestas de esta Comisión fue el de incentivar la corresponsabilidad de la madre y el padre en el cuidado de sus hijos e hijas. ¿Cree usted que el proyecto del Ejecutivo está bien orientado a cumplir con este objetivo?

MGC: Creo que hay un gran avance en ese sentido. La idea de la Comisión era más ambiciosa. En todos los países del mundo en que están más desarrolladas las políticas de familia, cuando nace un hijo o hija existen los permisos que son exclusivos para las madres, que nunca se extienden más de tres meses y después un permiso para la familia, en que la familia decide libremente cómo toma ese permiso y cómo se hace cargo del cuidado de sus hijos. Para nosotros, fue evidente que si el postnatal se iba a extender, debía ser un permiso para la familia, de manera de desligar un poco el cuidado de los hijos de la madre. En Chile está todavía asociado ciento por ciento a la mujer. También hay que considerar, y ese era nuestro argumento, que las personas que iban a decidir sobre la extensión del postnatal no son las personas que van a hacer uso del permiso. Las personas que lo van a usar son mucho más jóvenes y ya tienen más incorporada la idea de que los hombres participen en la crianza. Pero de todas formas, considerando nuestro estado de desarrollo cultural en esta materia, me parece que el proyecto recoge la idea de que el hombre tiene que empezar a participar.

VU: El padre solo puede tomarse 6 de las 12 semanas del permiso postnatal parental y no las 12 semanas completas. Además se toma como base para el cálculo del subsidio la remuneración de la mujer y no del hombre, que en la mayor parte de los casos es más alta, por la brecha salarial que existe entre hombres y mujeres.

MGC: En ese aspecto, es interesante que antes de implementar una medida así se miren las políticas públicas desde el punto de vista de los incentivos que se están poniendo detrás de cada medida. Si se calcula el subsidio tomando en cuenta la remuneración del padre hay mayor incentivo para que el padre se tome el permiso, porque va recibir un subsidio más alto que la madre. Esa era la idea de la Comisión.

VU: Que no se recogió en el proyecto de gobierno por razones presupuestarias.

MGC: Puede que esa haya sido la razón, pero yo no participé en la elaboración del proyecto, por lo que no conozco las motivaciones, sólo me las puedo imaginar.

VU: Pero el hecho de que el padre pueda tomarse solo 6 de las 12 semanas no tiene una justificación presupuestaria.

MGC: Ah, no. Yo pienso que detrás de eso está el hecho que incorporar esta medida en el proyecto no fue para nada fácil, porque muchos caballeros encuentran que es una locura. De verdad, a mí me llamó la atención, que muchos señores encontraban que era una locura pensar que los hombres se fueran a cuidar guaguas. ¡Están locas, cómo se les ocurre que lo van a hacer! Desgraciadamente existen también muchas mujeres que piensan que es una locura. 
VU: Y también podría haberse cambiado en el proyecto la forma en que se paga la licencia de 5 días que tiene el padre cuando nace un/a hijo/a. El subsidio de postnatal de la madre lo paga el Estado, pero estos 5 días del padre los paga el empleador.

MGC: Y nosotros propusimos que se cambiara, porque ahora el padre tiene que pedírselo al empleador y eso es muchas veces mal visto. No tengo una buena explicación fuera de posibles razones presupuestarias.

VU: Uno de los problemas del actual régimen de postnatal es su carácter regresivo, que se evidencia en el hecho de que el $52 \%$ del total de los recursos gastados en el subsidio maternal se destina a mujeres del quintil de más altos ingresos y solo el 5\% sobre el quintil de menores ingresos. ¿En qué medida cree usted que el proyecto del ejecutivo se hace cargo de este problema?

MGC: En que se baja el tope del subsidio a 30 UF.

\section{$V U:$ ¿Y es esa una medida apropiada?}

MGC: Yo creo. Hay dos maneras de hacerlo. Una es aumentar la cobertura del subsidio cambiando los requisitos y criterios de elegibilidad y la otra es bajar el tope. Esta última medida se basa en la idea de que las mujeres que tienen mayores sueldos están más capacitadas y tienen mayor poder de negociación con el empleador. Las mujeres que tienen buenos trabajos muchas veces, por acuerdos con el empleador, reciben la totalidad de su sueldo durante el postnatal. Entonces, se prioriza que el dinero del Estado alcance para más mujeres que ganan menos. Las 64 UF actuales equivalen a un millón y tanto, y el sueldo promedio de las mujeres es alrededor de \$500.000.

VU: Sí, se calcula que con el tope de 30 UF cerca del $80 \%$ de las mujeres que harían uso del postnatal parental recibirían un subsidio equivalente a su sueldo completo. Ahora, ¿qué piensa usted del argumento de que sería inconstitucional que las mujeres que ganan hasta 30 UF estén sometidas a un régimen rígido en que deben tomarse los 6 meses de postnatal y las que ganen más que eso tienen flexibilidad para volver a trabajar en forma parcial después de los primeros tres meses para no ver disminuida su remuneración?

MGC: En mi opinión y en la de la mayoría de los miembros de la Comisión, salvo dos de ellos, el periodo de extensión del postnatal debía poder ser tomado por todas las personas en forma flexible.

\section{VU: Quienes no estaban de acuerdo eran Ricardo Solari y María Elena Valenzuela.}

MGC: Sí. Su argumento era que los empleadores les iban a pasar por encima. Pero no era el argumento contra la flexibilidad en sí, sino en contra de la posibilidad de las mujeres de ejercer el derecho. Se ponía en duda que las mujeres pudieran tener capacidad negociadora para decirle al empleador que quieren tomarse el permiso.

VU: Hubo consenso en la Comisión, y me parece que también lo hay en la opinión pública, en que más que extender el postnatal a las personas que tienen derecho a él, es prioritario darle derecho a postnatal a más del $30 \%$ de las mujeres trabajadoras que no tienen acceso a éste. El proyecto del gobierno considera extender el beneficio a las mujeres que pertenezcan al $20 \%$ más pobre de la población, siempre que tengan doce meses de afiliación previsional antes del embarazo, al menos 8 cotizaciones en los 24 meses anteriores al embarazo y que su última cotización haya sido en virtud de un contrato a plazo fijo o por obra o faena. ¿Le parece que el proyecto recoge 
suficientemente la propuesta de la Comisión de extender el subsidio maternal a todas aquellas mujeres que han ejercido alguna actividad laboral acreditada durante un periodo mínimo en el año anterior a producirse el embarazo?

MGC: Una de las cosas que teníamos claras en la Comisión fue que en la ampliación de la cobertura del postnatal no nos podíamos olvidar de la importancia de los incentivos para lograr una mayor formalización del empleo femenino. Hay que tomar en consideración que actualmente todo empleo, pero particularmente el de las mujeres, es mucho más volátil y que, por lo tanto, la posibilidad de que las mujeres tengan acceso a este beneficio solo con contrato indefinido se restringe muchísimo, especialmente para las más vulnerables. En ese sentido yo encuentro buena la proposición del gobierno. Ahora, la cantidad de meses que había que exigir que hubieran trabajado para acceder al postnatal era un tema que había que estudiar mucho. Nosotros calculamos que si se pedía que la persona hubiera cotizado un mes durante el año anterior al embarazo llegábamos a una cobertura del $100 \%$ de las mujeres que habían trabajado formalmente y que habían cotizado para poder tener derecho a seguro de desempleo, para lo cual se necesita haber tenido un contrato formal, aunque fuera cortito.

$V U:$ Obviamente es deseable que la gente tenga acceso a un trabajo formal, pero ¿se puede lograr eso incentivando a las mujeres a que busquen un trabajo formal? ¿Hay trabajos formales disponibles para ellas? $¿ O$ es que las mujeres no optan por un trabajo formal porque no le ven beneficios al corto plazo?

MGC: El trabajo formal se incentiva de muchas formas. Este es solo un granito de arena. Pero creo que dar un subsidio maternal a las mujeres que tienen trabajos informales puede ser un retroceso, cuando tú estás tratando de fomentar la formalidad tanto por el lado de las empresas como de las propias mujeres. Además es importante que se entienda la idea de que éste no es un subsidio a la maternidad; la idea del subsidio es que se da en reemplazo de tu capacidad de generar un ingreso. Lo que es injusto es que mujeres que sí generan ingresos, pero en ese momento preciso no están trabajando, cuando tienen un hijo el Estado no las apoye.

VU: El proyecto de ley contempla que el fuero maternal comience a contabilizarse desde el inicio del embarazo hasta un año después del nacimiento y que en los contratos a plazo fijo o por obra el término del fuero coincida con el término del contrato. Esto representa una reducción del fuero maternal actualmente vigente que es contraria a la propuesta original de la Comisión, que sugirió mantener el plazo del fuero maternal y solo hacer ajustes respecto de contratos a plazo fijo y por obra o faena cuya duración fuera menor a 6 meses. ¿Cuál es su opinión sobre el proyecto del ejecutivo en este aspecto?

MGC: La Comisión propuso que el plazo se mantuviera inalterado respecto de lo que existe hoy. Es decir, que si se extendía el postnatal no se extendiera el fuero. Lo que yo sí creo que es importante es que en los contratos a plazo fijo o por obra o faena que duren menos de seis meses el fuero se extinga junto con el término del contrato. Yo creo que eso es de las pocas medidas que dentro de este tipo de materias de protección a la maternidad que puede ayudar a la empleabilidad de las mujeres. Esto recoge la realidad actual de la mayor movilidad o precariedad laboral, que son las dos caras de la misma moneda. Lo que nosotros propusimos fue asimilar estos contratos de menos de seis meses a los contratos por servicios transitorios, porque no me parece correcto que si tú vas a trabajar por un plazo corto te tengan que desaforar para poder terminar con el contrato de trabajo. La gracia de eso es que tú puedes contratar a una mujer embarazada por tres meses, a la que antes jamás habrías contratado. 
VU: ¿Qué opina de la manera en que el proyecto de ley enfrenta el tema del subsidio por enfermedad de hijo menor de un año? De acuerdo al proyecto del gobierno se distingue entre enfermedades gravísimas y graves -definidas en un decreto- y sólo las gravísimas van a recibir la totalidad del subsidio completo de acuerdo con la remuneración de la madre. Las enfermedades graves darán derecho a un subsidio equivalente a la remuneración de la madre con un tope de 30 UF y después se contempla un copago escalonado de acuerdo al tramo de ingreso de la madre.

MGC: A mí personalmente me parece bien. Nosotros solo propusimos que existiera un protocolo que permitiera distinguir las enfermedades realmente graves de las otras y que hubiese una mayor fiscalización, porque lo que existe ahora es un escándalo.

VU: ¿Y no habría sido mejor, como propuso la Comisión, simplemente mejorar el sistema de fiscalización de las licencias? A veces puede ser difícil distinguir, no es lo mismo tener una enfermedad gravísima en la mejor clínica privada...

MGC: Parece que no es tan difícil. Yo no soy una entendida, pero lo que decían los doctores que estaban en la Comisión es que el Auge ha avanzado mucho en la protocolización de las enfermedades y eso sirve también en el tema de las licencias. Cuando la enfermedad es gravísima, corresponde que se dé todo el subsidio, porque no se puede aumentar la carga para esa familia que ya lo está pasando muy mal, pero había que poner algún incentivo para terminar con el mal uso que se hace de las licencias. Mejorar el tema de la fiscalización, como nosotros propusimos, también va a demorar mucho tiempo y es difícil de implementar. Por eso entiendo que en el proyecto se dé este tratamiento más específico.

VU: Ésta es una pregunta un poco distinta. Cuando se discute una legislación de esta naturaleza, se manifiestan las distintas visiones respecto de los roles que deben cumplir las mujeres y los hombres en nuestra sociedad, tanto los asociados a la familia como los relacionados con el trabajo. Después de haber escuchado tantas opiniones en la Comisión y también a través de los medios de comunicación ¿cuál cree usted que son las visiones del hombre y de la mujer que predominan en este debate y cuáles están detrás del proyecto del Ejecutivo?

MGC: A mí lo que me llama la atención es que todavía existe una visión muy asociada a los roles y que se considera poco a las personas y sus intereses individuales. A mí me llamó mucho la atención un documento que leí hecho por el gobierno sueco, que era un estudio crítico de las políticas de familia en los países escandinavos. Ellos se cuestionan ¿por qué tenemos estas leyes? o ¿qué buscamos con esta reforma? Su respuesta es: queremos una sociedad en que cada persona sea capaz de valerse por sí misma, por lo tanto, cada persona tiene que tener acceso al trabajo. Eso produce una equidad o paridad de roles muy importante, que hace que cada persona pueda desarrollarse en plenitud. Eso no existe acá.

\section{VU: ¿Qué existe acá?}

MGC: Acá todavía las mujeres son sobre todo madres, los padres son sobre todo proveedores. Si las mujeres quieren salir a trabajar, perfecto, pero siguen siendo las únicas encargadas del cuidado y mi impresión es que eso produce un desequilibrio en la sociedad. Esta diferencia de roles se produce en parte por la naturaleza y en parte por el tipo de trabajo que existía antes. Desde el momento en que cambia el tipo de trabajo y las mujeres pueden salir a trabajar, se produce el desequilibrio de que las mujeres tienen más roles y los hombres se quedan con menos roles. Hay que hacer esfuerzos por modificar eso. 
VU: Me Ilama la atención, leyendo los comentarios en la prensa, que hay una especie de disputa sobre este proyecto, sobre si es un proyecto para los niños o es un proyecto para fomentar la empleabilidad de la mujer. Pareciera que fueran objetivos contradictorios, eso me produce mucha rebeldía.

MGC: A mí, además de rebeldía, me produce enojo, porque considero que abusan de la ignorancia del electorado con esos argumentos. ¿Qué es eso de que sea un proyecto para los niños y no para las mujeres? Si las mujeres que tienen un hijo hoy en día, la mayor parte lo tiene porque quiere tenerlo. Existen mediciones hechas en el extranjero que muestran que cuando las mujeres quieren trabajar y tener hijos, si se les hace muy difícil conciliar las dos cosas, dejan de tener hijos, no dejan de trabajar. Se dice que esto es por los hijos, ipero si para que haya hijos se necesita que haya mujeres que quieran tener hijos! Para que haya mujeres que quieran tener hijos hay que hacerles más fácil poder trabajar y quitarles un poco de encima las tareas de cuidado de la familia, o cuidado, en general.

\section{VU: Además, parece haber implícita una crítica a la mujer por ser egoísta ...}

MGC: Bueno, y por último si es así, y las mujeres (o la mayoría de las mujeres) somos egoístas porque queremos trabajar y ser madres, es lo que hay nomás. Entonces, hay que hacer políticas públicas que estén de acuerdo con los tiempos.

\section{VU: Además está el argumento de la baja tasa de natalidad.}

MGC: Pero es la realidad, el caso alemán es un ejemplo fantástico. A las alemanas les pagaron mucho para que dejaran de trabajar y se fueran a su casa a tener niños y no se fueron a su casa. La tasa de natalidad sigue bajando. Tienen que optar por trabajar o tener niños y optan por trabajar. Será espantoso o egoísta, pero es un dato.

VU: En cambio hay otros países en que ha subido la tasa de natalidad porque se han implementado políticas dirigidas a hacer compatible la maternidad y paternidad con el trabajo.

MGC: En ese sentido, lo que se ha aprendido de esas experiencias y de información recabada de las mismas mujeres, es que lo que más incidencia tiene en que las mujeres sigan teniendo hijos es que ellas sientan que si tienen un hijo no están completamente solas. Que el padre, el Estado, la sociedad comparten la responsabilidad. Que va a haber un padre, sala cuna, una red de apoyo, alguien que las ayude con esta tarea del cuidado. Si sienten que tienen el apoyo van a tener niños. Si sienten que están solas y que se tienen que hacer cargo ellas solas, aunque les paguen las ganas, no lo harán. 